\title{
A STABILIZED GALERKIN METHOD FOR A THIRD-ORDER EVOLUTIONARY PROBLEM
}

\author{
G. FAIRWEATHER, J. M. SANZ-SERNA, AND I. CHRISTIE
}

\begin{abstract}
The periodic initial value problem for the partial differential equation $u_{t}+u_{x x x}+\beta\left(u^{2}\right)_{x}+\frac{\gamma}{2}\left(u^{2}\right)_{x x}+\varepsilon u_{x x}-\delta u_{t x}=0, \varepsilon, \delta>0$, arises in fluidization models. The numerical integration of the problem is a difficult task in that many "reasonable" finite difference and finite element methods give rise to unstable discretizations. We show how to modify the standard Galerkin technique in order to stabilize it. Optimal-order error estimates are derived and the results of numerical experiments are presented. The stabilization technique suggested in the paper can be interpreted as rewriting the problem in Sobolev form and would also be useful for other equations involving terms of the form $u_{t}-\delta u_{t x}$.
\end{abstract}

\section{INTRODUCTION}

The periodic initial value problem for the real-valued function $u(x, t)$ given by

$$
\begin{gathered}
u_{t}+u_{x x x}+\beta\left(u^{2}\right)_{x}+\frac{\gamma}{2}\left(u^{2}\right)_{x x}+\varepsilon u_{x x}-\delta u_{t x}=0, \quad x \in \mathbb{R}, t \in[0, T] \\
u(x+2 \pi, t)=u(x, t), \quad x \in \mathbb{R}, t \in[0, T] \\
u(x, 0)=q(x), \quad x \in \mathbb{R},
\end{gathered}
$$

where $\beta, \gamma, \varepsilon, \delta$ are real constants, $\varepsilon, \delta>0$, arises in the modelling of flow in a fluidized bed (Ganser and Drew [7, 8]). The unknown $u$ represents the value of a spatially periodic small perturbation of the concentration of particles. Christie and Ganser [3] found that the numerical integration of (1.1)-(1.3) presents unexpected difficulties. In fact, several "reasonable" time-implicit, finite difference and Galerkin schemes are unconditionally unstable, while other

Received May 18, 1989.

1980 Mathematics Subject Classification (1985 Revision). Primary 65M60; Secondary 65M15.

Key words and phrases. Stability, Galerkin methods, fluidized beds, Sobolev equations.

The first author was supported in part by the National Science Foundation (Grant No. RII8610671) and the Commonwealth of Kentucky through the Kentucky EPSCoR Program, and by the Commission for Educational Exchange Between the United States of America and Spain while a Fulbright Scholar at the Universidad de Valladolid, Valladolid, Spain.

The second author was supported in part by Fondo Nacional para el Desarrollo de la Investigacion Cientifica y Tecnica, Project PB-86-0313.

The third author was supported in part by the Energy and Water Research Center of West Virginia University and by the U. S. Department of Energy, Project DE-FG05-88ER25067. 
schemes cannot be used with small values of the time step. The origin of these strange phenomena has been studied by Abia et al. [1]. It is expedient to summarize briefly their findings. First of all, the instabilities are invariably due to the space discretization. This implies that the difficulties encountered by Christie and Ganser cannot be circumvented by using a more sophisticated time integrator such as an automatic package. Second, the instabilities essentially arise from the terms $u_{t}+\varepsilon u_{x x}-\delta u_{t x}$, so that to gain some understanding of the situation it is sufficient to consider the equation

$$
u_{t}+\varepsilon u_{x x}-\delta u_{t x}=0, \quad \varepsilon, \delta>0 .
$$

Fourier analysis shows that, at low wave numbers, the backward heat equation combination $u_{t}+\varepsilon u_{x x}$ dominates, and therefore the corresponding Fourier modes grow exponentially. This growth is linked to the development of slugs in the fluidization process. However, at high wave numbers, the term involving $u_{t x}$ offsets the importance of the term $u_{t}$ and introduces a stabilizing mechanism which results in the $L^{2}$-well-posedness of (1.2)-(1.4). Unfortunately, most standard spatial discretizations of (1.4) are unable to deal with the $u_{t x}$ term in such a way that the stabilizing properties are not lost in the discretization procedure. For example, the standard Galerkin technique based on piecewise linear test/trial functions on a uniform grid leads to the system of ordinary differential equations

$$
\frac{1}{6} \dot{U}_{j+1}+\frac{2}{3} \dot{U}_{j}+\frac{1}{6} \dot{U}_{j-1}+\varepsilon \frac{U_{j+1}-2 U_{j}+U_{j-1}}{h^{2}}-\delta \frac{\dot{U}_{j+1}-\dot{U}_{j-1}}{2 h}=0,
$$

where a dot denotes differentiation with respect to $t$. When saw-tooth mode solutions of the form $U_{j}(t)=(-1)^{j} f(t)$ are sought, substitution in (1.5) yields

$$
\frac{1}{3} \frac{d f}{d t}-4 \varepsilon h^{-2} f=0
$$

that is,

$$
f(t)=\exp \left[12 \varepsilon h^{-2} t\right] f(0) .
$$

Thus, as $h \rightarrow 0,(1.5)$ possesses solutions which grow exponentially at arbitrarily fast rates and, therefore, (1.5) is unstable.

The situation just described contradicts an apparently widely-held belief that Galerkin discretizations of well-posed evolutionary problems are automatically stable. The purpose of this paper is to show how to modify the standard Galerkin approach so as to discretize (1.1) and related equations in a stable manner. The new method suggested in this paper is, in some respects, similar to Galerkin methods for first-order hyperbolic problems proposed by Dendy [5] and Wahlbin [10,11], and the Galerkin method for the Korteweg-de Vries equation discussed by Wahlbin [11]. Note, however, that these authors were motivated by the suboptimality of the convergence rate of the standard Galerkin approach in the problem that they were considering, while here the standard 
Galerkin procedure has to be modified in the first place to obtain a convergent method.

A brief outline of this paper is as follows. Section 2 contains a description of the new technique. In $\S 3$, we prove that the proposed procedure is, in fact, convergent. Furthermore, the rate of convergence is optimal. Section 4 is devoted to numerical experiments and the final section, $\S 5$, contains some concluding remarks.

\section{A MODIFIED GALERKIN METHOD}

We first introduce some notation. The functions considered are real-valued. On the space $L^{2}(I), I=(0,2 \pi)$, let $(\cdot, \cdot)$ and $\|\cdot\|$ denote the standard inner product and norm, respectively. For any integer $m \geq 0, H^{m}$ denotes the Sobolev space of $2 \pi$-periodic functions on $\mathbb{R}$ with $m$ derivatives in $L_{\text {loc }}^{2}(\mathbb{R})$, where the associated norm is given by

$$
\|v\|_{m}=\left(\sum_{j=0}^{m}\left\|\frac{d^{j} v}{d x^{j}}\right\|^{2}\right)^{1 / 2}
$$

Note that, for $u \in H^{2}$,

$$
\left\|u^{\prime}\right\|^{2}=\int_{0}^{2 \pi} u^{\prime} u^{\prime} d x=-\int_{0}^{2 \pi} u u^{\prime \prime} d x \leq\|u\|\left\|u^{\prime \prime}\right\|,
$$

where the prime denotes differentiation with respect to $x$. Clearly, (2.1) shows that

$$
\left\|u^{\prime}\right\|^{2} \leq \frac{1}{2}\left(\|u\|^{2}+\left\|u^{\prime \prime}\right\|^{2}\right)
$$

hence $\sqrt{\|u\|^{2}+\left\|u^{\prime \prime}\right\|^{2}}$ is a norm on $H^{2}$ equivalent to $\|\cdot\|_{2}$.

Let $L^{\infty}(I)$ denote the space of functions $v$ on $I$ such that

$$
\|v\|_{L^{\infty}}=\operatorname{ess} \sup |v(x)|<\infty .
$$

If $X$ is a normed space with norm $\|\cdot\|_{X}$ and $v:[0, T] \rightarrow X$, then

$$
\|v\|_{L^{2}(X)}=\left[\int_{0}^{T}\|v(t)\|_{X}^{2} d t\right]^{1 / 2} \quad \text { and }\|v\|_{L^{\infty}(X)}=\underset{t \in[0, T]}{\operatorname{ess} \sup }\|v(t)\|_{X} .
$$

The piecewise polynomial spaces used in the treatment of (1.1) are defined in the following way. Let $\Delta=\left\{x_{0}, x_{1}, \ldots, x_{N}\right\}$ denote a partition of $\bar{I}$, where $0=x_{0}<x_{1}<\cdots<x_{N}=2 \pi$. Let $\bar{I}_{k}=\left[x_{k-1}, x_{k}\right], h_{k}=x_{k}-x_{k-1}$, $k=1,2, \ldots, N$, and $h=\max _{k} h_{k}$. For a closed interval $E$, let $P_{r}(E)$ denote the set of polynomials on $E$ of degree at most $r$. Then the piecewise polynomial spaces we shall use are

$$
S_{\Delta}=\left\{v \mid v \in H^{2}, v \in P_{r}\left(\bar{I}_{k}\right), k=1,2, \ldots, N\right\}
$$


where $r \geq 3$ is a fixed integer. It is well known that such spaces have the property that there exists a constant $C$, independent of $\Delta$, such that, for $u \in$ $H^{r+1}$,

$$
\inf _{v \in S_{\Delta}}\|u-v\|_{H^{i}} \leq C h^{j-i}\|u\|_{H^{j}},
$$

with $i=0,1,2$ and $i \leq j \leq r+1$. In fact, in what follows, the spaces $S_{\Delta}$ in (2.2) may be replaced by any other subspace of $H^{2}$ for which (2.3) holds.

The weak form of the initial value problem (1.1) on which the new semidiscrete Galerkin method is based is derived in the following way. We first take the inner product of $(1.1)$ and the test functions $v-\delta v_{x}$, with $v \in H^{2}$ arbitrary, to obtain

$$
\left(u_{t}+u_{x x x}+\beta\left(u^{2}\right)_{x}+\frac{\gamma}{2}\left(u^{2}\right)_{x x}+\varepsilon u_{x x}-\delta u_{t x}, v-\delta v_{x}\right)=0,
$$

or

$$
\begin{aligned}
& \left(u_{t}, v\right)-\delta\left(u_{t}, v_{x}\right)+\left(u_{x x x}, v-\delta v_{x}\right)+2 \beta\left(u u_{x}, v-\delta v_{x}\right) \\
& \quad+\gamma\left(\left(u u_{x}\right)_{x}, v-\delta v_{x}\right)+\varepsilon\left(u_{x x}, v-\delta v_{x}\right)-\delta\left(u_{t x}, v\right)+\delta^{2}\left(u_{t x}, v_{x}\right)=0 .
\end{aligned}
$$

Then we integrate the second, third, fifth, and sixth terms by parts and simplify to obtain the required weak form of (1.1), namely

$$
\begin{aligned}
& \left(u_{t}, v\right)-\left(u_{x x}, v_{x}-\delta v_{x x}\right)+2 \beta\left(u u_{x}, v-\delta v_{x}\right) \\
& -\gamma\left(u u_{x}, v_{x}-\delta v_{x x}\right)-\varepsilon\left(u_{x}, v_{x}-\delta v_{x x}\right)+\delta^{2}\left(u_{t x}, v_{x}\right)=0, \quad \text { for all } v \in H^{2} .
\end{aligned}
$$

Note that all inner products appearing make sense if, for $t \in[0, T], u(t) \in H^{2}$ and $u_{t}(t) \in H^{1}$.

The semidiscrete method for (1.1)-(1.3) consists in seeking $U:[0, T] \rightarrow S_{\Delta}$ such that $U(0)$ is an approximation to the initial datum $q$ and

$$
\begin{gathered}
\left(U_{t}, v\right)-\left(U_{x x}, v_{x}-\delta v_{x x}\right)+2 \beta\left(U U_{x}, v-\delta v_{x}\right)-\gamma\left(U U_{x}, v_{x}-\delta v_{x x}\right) \\
-\varepsilon\left(U_{x}, v_{x}-\delta v_{x x}\right)+\delta^{2}\left(U_{t x}, v_{x}\right)=0, \quad \text { for all } v \in S_{\Delta} .
\end{gathered}
$$

Note that if $\left\{\Phi_{j}\right\}$ is a basis for $S_{\Delta}$ and $U(t)=\sum_{j} \alpha_{j}(t) \Phi_{j}$, then (2.4) is equivalent to a system of ordinary differential equations,

$$
M \dot{\boldsymbol{\alpha}}=\mathbf{F}(\boldsymbol{\alpha}),
$$

where $\boldsymbol{\alpha}$ is the vector of unknown coefficients $\left\{\alpha_{j}\right\}, \mathbf{F}$ is a nonlinear function, and $M$ is the matrix with entries $\left(\Phi_{i}, \Phi_{j}\right)+\delta^{2}\left(\Phi_{i}^{\prime}, \Phi_{j}^{\prime}\right)$. Since $M$ is the Gram matrix of the basis $\left\{\Phi_{j}\right\}$ with respect to the inner product $[\phi, \psi]=$ $(\phi, \psi)+\delta^{2}\left(\phi^{\prime}, \psi^{\prime}\right)$, it follows that $M$ is nonsingular. Thus (2.4) possesses a unique solution defined, at least, for $t \in\left[0, t_{\max }\right), t_{\max }=t_{\max }(h)$.

\section{ERRor ESTIMATES}

In order to estimate the accuracy of the semidiscrete approximation $U$, it is necessary to introduce a suitable elliptic projection $W$ of the solution $u$ of 
(1.1)-(1.3). To define $W$, we require additional notation. Let $B=B_{c_{1}, c_{2}, c_{3}}$ denote the bilinear form on $H^{2} \times H^{2}$ defined by

$$
\begin{aligned}
B(\phi, \psi)= & \delta\left(\phi^{\prime \prime}, \psi^{\prime \prime}\right)+\left(c_{3} \phi^{\prime}, \psi^{\prime \prime}\right)+\left(c_{2} \phi^{\prime}, \psi^{\prime}\right) \\
& +\left(c_{1} \phi^{\prime}, \psi\right)+K(\phi, \psi), \quad \text { for all } \phi, \psi \in H^{2},
\end{aligned}
$$

where $c_{1}, c_{2}, c_{3}$ are bounded $2 \pi$-periodic functions of $x$, and $K$ is a suffciently large positive constant whose choice will be discussed momentarily.

Clearly, $B$ is continuous; that is, there exists a constant $C$ such that, for all $\phi, \psi \in H^{2}$,

$$
|B(\phi, \psi)| \leq C\|\phi\|_{H^{2}}\|\psi\|_{H^{2}} .
$$

Furthermore, note that $C$ only depends on $\delta, K$, and $\left\|c_{i}\right\|_{L^{\infty}}, i=1,2,3$. On the other hand, since (2.1) implies

$$
\left\|u^{\prime}\right\|^{2} \leq \frac{1}{2 b}\left\|u^{\prime \prime}\right\|^{2}+\frac{b}{2}\|u\|^{2},
$$

where $b$ is an arbitrarily large positive constant, it is easy to show that $K$ can be chosen sufficiently large that

$$
B(\phi, \phi) \geq \frac{\delta}{2}\left\{\left\|\phi^{\prime \prime}\right\|^{2}+\|\phi\|^{2}\right\}, \quad \phi \in H^{2} ;
$$

that is, $B$ is coercive in $H^{2}$. The size of $K$ depends only on $\delta$ and $\left\|c_{i}\right\|_{L^{\infty}}$, $i=1,2,3$.

We now define $W:[0, T] \rightarrow S_{\Delta}$ by

$$
B(W(t), v)=B(u(t), v), \quad v \in S_{\Delta}, t \in[0, T],
$$

where, for each $t \in[0, T]$, the functions $c_{i}, i=1,2,3$, in (3.1) are chosen as

$$
\begin{aligned}
& c_{3}(\cdot, t)=1+\varepsilon \delta+\gamma \delta u(\cdot, t), \\
& c_{2}(\cdot, t)=-2 \beta \delta u(\cdot, t)-\gamma u(\cdot, t)-\varepsilon, \\
& c_{1}(\cdot, t)=2 \beta u(\cdot, t) .
\end{aligned}
$$

Under the assumption that $u(\cdot, t) \in H^{2}$ for $t \in[0, T]$ (necessary for $u$ to be a weak solution), the functions $c_{i}, i=1,2,3$, in (3.5) are in $L^{\infty}(I)$. Therefore, (3.2) and (3.3) hold, and the Lax-Milgram theorem (Ciarlet [4]) implies that $W$ is uniquely defined.

Theorem 3.1. Assume $u, u_{t} \in L^{\infty}\left(H^{r+1}\right), r \geq 3$. Then there exists a constant $C$, depending on $u, \beta, \gamma, \delta, \varepsilon, T, r$ but independent of $t$ and of the partition $\Delta$, such that

$$
\left\|\frac{\partial^{l}}{\partial t^{l}}(u-W)(t)\right\|_{H^{\prime}} \leq C h^{r+1-i}, \quad l=0,1, i=0,1,2, t \in[0, T] .
$$

Proof. We first note that $u \in L^{\infty}\left(H^{r+1}\right)$ implies $u \in L^{\infty}\left(L^{\infty}\right)$, via the Sobolev embedding theorem. Therefore, the bilinear form $B$ defined by (3.1) and (3.5) 
is continuous and coercive uniformly in $t \in[0, T]$, and the estimate (3.6), with $l=0, i=2$ follows from the approximation property (2.3) in a standard way.

Next we address the case $l=0, i=0$ by a Nitsche argument. For $t \in$ $[0, T]$, we set $\eta(t)=u(t)-W(t) \in H^{2}$ and define $\phi(t) \in H^{2}$ by

$$
B(v, \phi(t))=(v, \eta(t)) \text { for all } v \in H^{2} .
$$

By applying the Lax-Milgram theorem to $B^{*}$, where $B^{*}(\phi, \psi)=B(\psi, \phi)$, $\psi, \phi \in H^{2}$, it follows that $\phi$ is uniquely defined. Integration by parts in (3.7) reveals that $\phi$ satisfies, in the sense of distributions,

$$
\delta \phi_{x x x x}-\left(c_{3} \phi_{x x}\right)_{x}-\left(c_{2} \phi_{x}\right)_{x}-\left(c_{1} \phi\right)_{x}+K \phi=\eta .
$$

Since $u_{x} \in L^{\infty}\left(L^{\infty}\right)$ and therefore $c_{i} \in L^{\infty}\left(L^{\infty}\right), i=1,2,3$, it follows by standard techniques that $\eta \in L^{2}$ implies that $\phi \in H^{4}$ and

$$
\|\phi(t)\|_{H^{4}} \leq C\|\eta(t)\|_{L^{2}}, \quad t \in[0, T],
$$

where $C$ depends on $\delta,\left\|c_{i}\right\|_{L^{\infty}\left(L^{\infty}\right)}$, and $\left\|\left(c_{i}\right)_{x}\right\|_{L^{\infty}\left(L^{\infty}\right)}$. Then, for $\chi \in S_{\Delta}$, we have, from (3.7), (3.4), and (3.2),

$$
\|\eta\|^{2}=B(\eta, \eta)=B(\eta, \phi)=B(\eta, \phi-\chi) \leq C\|\eta\|_{H^{2}} \cdot\|\phi-\chi\|_{H^{2}} .
$$

From (2.3), $\chi \in S_{\Delta}$ can be chosen such that

$$
\|\phi-\chi\|_{H^{2}} \leq C h^{2}\|\phi\|_{H^{4}}
$$

On combining (3.8), (3.9), (3.10), it follows that

$$
\|\eta\| \leq C h^{2}\|\eta\|_{H^{2}}
$$

which implies (3.6) with $l=0, i=0$. The case $l=0, i=1$ follows from interpolation between the cases $l=0, i=0$, and $l=0, i=2$.

To prove (3.6) for $l=1$, we must differentiate (3.7) to obtain, for $\chi \in S_{\Delta}$,

$$
B\left(\eta_{t}, \chi\right)=\left(\hat{c}_{3} \eta_{x}, \chi_{x x}\right)+\left(\hat{c}_{2} \eta_{x}, \chi_{x}\right)+\left(\hat{c}_{1} \eta_{x}, \chi\right),
$$

where

$$
\hat{c}_{3}=-\gamma \delta u_{t}, \quad \hat{c}_{2}=(2 \beta \delta+\gamma) u_{t}, \quad \hat{c}_{1}=-2 \beta u_{t} .
$$

Since $u_{t} \in L^{\infty}\left(L^{\infty}\right)$, it follows that $\hat{c}_{1}, \hat{c}_{2}, \hat{c}_{3} \in L^{\infty}\left(L^{\infty}\right)$. Then, using (3.3) and (3.2), we can write, for $\chi \in S_{\Delta}$,

$$
\begin{aligned}
\frac{\delta}{2}\left(\left\|\eta_{x x t}\right\|^{2}+\left\|\eta_{t}\right\|^{2}\right) \leq & B\left(\eta_{t}, \eta_{t}\right)=B\left(\eta_{t}, u_{t}-\chi\right)+B\left(\eta_{t}, \chi-W_{t}\right) \\
\leq & C\left\{\left\|\eta_{x x t}\right\|+\left\|\eta_{t}\right\|\right\}\left\|u_{t}-\chi\right\|_{H^{2}}+\left(\hat{c}_{3} \eta_{x},\left(\chi-W_{t}\right)_{x x}\right) \\
& +\left(\hat{c}_{2} \eta_{x},\left(\chi-W_{t}\right)_{x}\right)+\left(\hat{c}_{1} \eta_{x},\left(\chi-W_{t}\right)\right) \\
\leq & C\left[\left\{\left\|\eta_{x_{x x}}\right\|+\left\|\eta_{t}\right\|\right\}\left\|u_{t}-\chi\right\|_{H^{2}}+\|\eta\|_{H^{1}} \cdot\left\|\chi-W_{t}\right\|_{H^{2}}\right] \\
\leq & \left(\left\|\eta_{x x t}\right\|^{2}+\frac{\delta}{4}\left\|\eta_{t}\right\|^{2}\right)+C\left[\left\|u_{t}-\chi\right\|_{H^{2}}^{2}+\|\eta\|_{H^{1}}^{2}\right] .
\end{aligned}
$$


In view of the approximation properties of $S_{\Delta}$, these estimates prove the case $l=1, i=2$ in (3.6). To prove (3.6) with $l=1, i=0$, techniques similar to those developed by Dupont et al. [6] can be used. Finally, the case $l=1$, $i=1$ follows from interpolation.

We are now in a position to provide error bounds for the solution $U$ of (2.4).

Theorem 3.2. Assume that $u, u_{t} \in L^{\infty}\left(H^{r+1}\right), r \geq 3$, and that $U(0)$ is chosen to be the projection $W(0)$ of $u(0)$. Then, for $h$ sufficiently small, the solution $U$ of (2.4) exists for $t \in[0, T]$ and

(3.12) $\|u-U\|_{L^{\infty}\left(L^{2}\right)}+h\|u-U\|_{L^{\infty}\left(H^{1}\right)}+h^{2}\|u-U\|_{L^{2}\left(H^{2}\right)}=O\left(h^{r+1}\right)$ as $h \rightarrow 0$.

Proof. Since $L^{\infty}\left(H^{1}\right) \subset L^{\infty}\left(L^{\infty}\right)$ with continuous imbedding, standard arguments (see, for example, Wahlbin [11]) can be invoked to show that we need only prove that (3.12) holds under the assumptions that $U$ exists up to $t=T$ and that $U \in L^{\infty}\left(L^{\infty}\right)$. As before, we set $\eta=u-W$. In view of the estimate (3.6) for $\eta$, it is sufficient to show that $\zeta=U-W$ satisfies

$$
\|\zeta\|_{L^{\infty}\left(L^{2}\right)}+\|\zeta\|_{L^{\infty}\left(H^{1}\right)}+\|\zeta\|_{L^{2}\left(H^{2}\right)}=O\left(h^{r+1}\right) .
$$

A simple calculation establishes that, if $v \in S_{\Delta}$,

$$
\begin{aligned}
\left(\zeta_{t}, v\right) & -\left(\zeta_{x x}, v_{x}-\delta v_{x x}\right)+2 \beta\left(U \zeta_{x}+W_{x} \zeta, v-\delta v_{x}\right) \\
& -\gamma\left(U \zeta_{x}+W_{x} \zeta, v_{x}-\delta v_{x x}\right)-\varepsilon\left(\zeta_{x}, v_{x}-\delta v_{x x}\right)+\delta^{2}\left(\zeta_{t x}, v_{x}\right) \\
= & \left(\eta_{t}, v\right)+2 \beta\left(W_{x} \eta, v-\delta v_{x}\right) \\
& -\gamma\left(W_{x} \eta, v_{x}-\delta v_{x x}\right)+\delta^{2}\left(\eta_{t x}, v_{x}\right)-K(\eta, v) .
\end{aligned}
$$

On setting $v=\zeta$ in this equality, we obtain

$$
\begin{aligned}
\frac{1}{2} \frac{d}{d t}\|\zeta\|^{2}+\delta\left\|\zeta_{x x}\right\|^{2}-\left(\zeta_{x x}, \zeta_{x}\right)+\frac{\delta^{2}}{2} \frac{d}{d t}\left\|\zeta_{x}\right\|^{2} \\
=\varepsilon\left\|\zeta_{x}\right\|^{2}+\varepsilon \delta\left(\zeta_{x}, \zeta_{x x}\right)+\gamma\left(U \zeta_{x}+W_{x} \zeta, \zeta_{x}-\delta \zeta_{x x}\right) \\
\quad-2 \beta\left(U \zeta_{x}+W_{x} \zeta, \zeta-\delta \zeta_{x}\right)-\gamma\left(W_{x} \eta, \zeta_{x}-\delta \zeta_{x x}\right) \\
\quad+\delta^{2}\left(\eta_{t x}, \zeta_{x}\right)-K(\eta, \zeta) .
\end{aligned}
$$

Now, by periodicity,

$$
\left(\zeta_{x x}, \zeta_{x}\right)=0
$$

The boundedness of $U$ assumed a priori, and the boundedness of $W_{x}$ implied by (3.6), enable us to write

$$
\begin{gathered}
\gamma\left(U \zeta_{x}+W_{x} \zeta, \zeta_{x}-\delta \zeta_{x x}\right) \leq C\left(\|\zeta\|^{2}+\left\|\zeta_{x}\right\|^{2}\right)+\frac{\delta}{6}\left\|\zeta_{x x}\right\|^{2} \\
2 \beta\left(U \zeta_{x}+W_{x} \zeta, \zeta-\delta \zeta_{x}\right) \leq C\left(\|\zeta\|^{2}+\left\|\zeta_{x}\right\|^{2}\right)
\end{gathered}
$$

and

$$
\gamma\left(W_{x} \eta, \zeta_{x}-\delta \zeta_{x x}\right) \leq C\left(\|\eta\|^{2}+\left\|\zeta_{x}\right\|^{2}\right)+\frac{\delta}{6}\left\|\zeta_{x x}\right\|^{2}
$$


Moreover,

$$
\left(\eta_{t x}, \zeta_{x}\right)=-\left(\eta_{t}, \zeta_{x x}\right) \leq C\left\|\eta_{t}\right\|^{2}+\frac{\delta}{6}\left\|\zeta_{x x}\right\|^{2}
$$

and

$$
-K(\eta, \zeta) \leq C\left(\|\eta\|^{2}+\|\zeta\|^{2}\right) .
$$

On substituting (3.15)-(3.20) in (3.14) and simplifying, we obtain

$$
\frac{d}{d t}\left(\|\zeta\|^{2}+\left\|\zeta_{x}\right\|^{2}\right)+\frac{\delta}{2}\left\|\zeta_{x x}\right\|^{2} \leq C\left[\|\zeta\|^{2}+\left\|\zeta_{x}\right\|^{2}+\|\eta\|^{2}+\left\|\eta_{t}\right\|^{2}\right] .
$$

Now Gronwall's lemma leads to (3.13) and the proof is complete.

Finally, we show that the Galerkin technique discussed in this paper does in fact overcome the stability problems described in the introduction. As was pointed out there, the instabilities stem from the terms present in the linear model (1.4), so that it is sufficient to analyze our technique when applied to this model, or equivalently to consider (1.1) with $\beta=\gamma=0$.

Theorem 3.3. When $\beta=\gamma=0$, the Galerkin solution defined in (2.4) satisfies the $H^{1}$ Lax-stability estimate

$$
\|U(t)\|^{2}+\delta^{2}\left\|U_{x}(t)\right\|^{2} \leq \exp \left(2 \varepsilon t / \delta^{2}\right)\left\{\|U(0)\|^{2}+\delta^{2}\left\|U_{x}(0)\right\|^{2}\right\}, \quad t \geq 0 .
$$

Proof. The proof follows easily by choosing $v=U$ in (2.4) and applying the energy method as in the proof of Theorem 3.2.

\section{NuMERicAl RESUltS}

Problem (1.1)-(1.3) was solved numerically by selecting $S_{\Delta}$ in (2.4) to be the space of periodic piecewise Hermite cubic polynomials on a uniform partition of $\bar{I}$. As in Abia et al. [1], the parameter values in (1.1) were taken to be $\beta=-0.45, \gamma=0.37947, \delta=0.04216, \varepsilon=0.09487$, and the initial condition was $q(x)=0.1 \sin x$.

Numerical experiments showed that the constant $K$ in (3.1) could be chosen such that the nodal values of the projection $W(0)$ in Theorem 3.2 and its derivative $W^{\prime}(0)$ were essentially identical to the nodal values of $q$ and its derivative $q^{\prime}$, respectively. It was decided therefore to take the interpolant of $q(x)$ as the initial condition for the system of ordinary differential equations resulting from (2.4). This system was then integrated in time by means of the trapezoidal rule with a small fixed time step. Newton's method was used to solve the system of nonlinear equations arising at each time step. The initial estimate of the solution was taken to be that computed at the previous time level, and the Jacobian, which is periodic block tridiagonal, was updated at each iteration. The iterative process was continued until the maximum norm of the difference between successive iterates was less than $10^{-12}$

As a comparison, we ran the second-order finite difference method suggested by Abia et al. [1], also using the trapezoidal rule to advance the solution in 
time. While this scheme only computes nodal approximations to $u$, secondorder approximations to $u^{\prime}$ at the nodes are easily determined by standard central differencing (see [1, Theorem 3.1]).

In Table 1, we present approximations to $u$ and $u^{\prime}$ at the point $x=0$, $t=1$ computed using (a) the new Galerkin method with Hermite cubics, and (b) the method of Abia et al., with $\Delta t=0.001$ in each case. The Hermite cubic approximations to $u^{\prime}(0,1)$ display an $O\left(h^{4}\right)$ behavior, while the approximations to $u(0,1)$ and $u^{\prime}(0,1)$ computed with the method of Abia et al. exhibit $O\left(h^{2}\right)$ convergence. Note that the Hermite approximation to $u(0,1)$ is highly accurate even on the coarsest partitions. Since each method requires a similar amount of computational effort, namely, the solution of periodic pentadiagonal linear systems in the method of Abia et al., and periodic block tridiagonal systems with $2 \times 2$ blocks in the case of the Galerkin method, the Galerkin method should clearly be preferred.

TABLE 1

Approximations to $u(0,1)$ and $u^{\prime}(0,1)$ computed using (a) the new Galerkin method; (b) the method of Abia et al. [1]

\begin{tabular}{|l|ll|lr|}
\hline & \multicolumn{2}{|c|}{$(\mathrm{a})$} & \multicolumn{2}{c|}{$(\mathrm{b})$} \\
$h$ & $u(0,1)$ & $u^{\prime}(0,1)$ & $u(0,1)$ & $u^{\prime}(0,1)$ \\
\hline 0.1 & $8.863513 \mathrm{E}-2$ & $5.572576 \mathrm{E}-2$ & $8.2864 \mathrm{E}-2$ & $5.7047 \mathrm{E}-2$ \\
0.05 & $8.864256 \mathrm{E}-2$ & $5.585955 \mathrm{E}-2$ & $8.6747 \mathrm{E}-2$ & $5.6228 \mathrm{E}-2$ \\
0.025 & $8.864264 \mathrm{E}-2$ & $5.588262 \mathrm{E}-2$ & $8.8146 \mathrm{E}-2$ & $5.6049 \mathrm{E}-2$ \\
0.0125 & $8.864264 \mathrm{E}-2$ & $5.588423 \mathrm{E}-2$ & $8.8517 \mathrm{E}-2$ & $5.5729 \mathrm{E}-2$ \\
0.00625 & $8.864264 \mathrm{E}-2$ & $5.588433 \mathrm{E}-2$ & $8.8611 \mathrm{E}-2$ & $5.5896 \mathrm{E}-2$ \\
\hline
\end{tabular}

It should be mentioned that we also conducted numerical experiments with fixed values of $h$ and decreasing sequences of $\Delta t$. As predicted, no instability was encountered.

\section{CONCLUding Remarks}

Equation (1.1) is of the form

$$
u_{t}-\delta u_{t x}=L(u)
$$

with $L$ a nonlinear differential operator which does not involve derivatives with respect to $t$. The method suggested in this paper is based on the weak form

$$
\left(u_{t}-\delta u_{t x}, v-\delta v_{x}\right)=\left(L(u), v-\delta v_{x}\right) .
$$

A formal integration by parts in (5.2) yields

$$
\left(u_{t}-\delta u_{t x}+\delta u_{t x}-\delta^{2} u_{t x x}, v\right)=\left(L(u)+\delta(L(u))_{x}, v\right),
$$

a weak form of the equation

$$
u_{t}-\delta^{2} u_{t x x}=L(u)+\delta(L(u))_{x} .
$$


In turn, (5.4) can be obtained by differentiating (5.1) to get

$$
u_{t x}-\delta u_{t x x}=(L(u))_{x}
$$

and then eliminating $u_{t x}$ between (5.1) and (5.5). Therefore, the novel weak form (5.2) of (5.1), based on the use of the somewhat artificial test functions $v-\delta v_{x}$, could have been obtained alternatively by (i) manipulating the strong form (5.1) to eliminate $u_{t x}$ and arrive at (5.4), (ii) forming the familiar weak form (5.3) of (5.4), and (iii) integrating by parts to obtain (5.2).

The observation which we have just made may be useful in other contexts. For instance, suppose we wish to discretize (1.1) by finite differences, and for simplicity let us suppose that we are dealing with the linear case $\beta=\gamma=0$. The discretization of (1.1) based on the standard centered replacements of $\partial / \partial x$, $\partial^{2} / \partial x^{2}, \partial^{3} / \partial x^{3}$ is unstable (Abia et al. [1]). On the other hand, if we first go to the form (5.4), which in the linear case becomes

$$
u_{t}-\delta^{2} u_{t x x}+\varepsilon u_{x x}+(1+\varepsilon \delta) u_{x x x}+\delta u_{x x x x}=0 \text {, }
$$

and then perform standard central differencing, we obtain a stable semidiscrete scheme. An alternative way of constructing stable finite difference schemes for (1.1) has been suggested by Abia et al., but their construction is perhaps of a more ad hoc nature than the one we have just presented.

It appears that the combination $u_{t}-\delta^{2} u_{t x x}$ in (5.4) is more easily discretized than the combination $u_{t}-\delta u_{t x}$ in the original form (5.1). It is perhaps useful to emphasize that the combination $u_{t}-\delta^{2} u_{t x x}$ appears in the so-called Sobolev equations $[2,9]$.

\section{BIBLIOGRAPHY}

1. L. Abia, I. Christie, and J. M. Sanz-Serna, Stability of schemes for the numerical treatment of an equation modelling fluidized beds, RAIRO Numer. Anal. Math. Modell. 23 (1989), 125-138.

2. D. N. Arnold, J. Douglas, Jr., and V. Thomée, Superconvergence of a finite element approximation to the solution of a Sobolev equation in a single space variable, Math. Comp. 36 (1981), 53-63.

3. I. Christie and G. Ganser, A numerical study of nonlinear waves arising in a one-dimensional model of a fluidized bed, J. Comput. Phys. 81 (1989), 300-318.

4. P. G. Ciarlet, The finite element method for elliptic problems, North-Holland, Amsterdam, 1978.

5. J. E. Dendy, Jr., Two methods of Galerkin type achieving optimum $L^{2}$ rates of convergence for first order hyperbolics, SIAM J. Numer. Anal. 11 (1974), 637-653.

6. T. Dupont, G. Fairweather, and J. P. Johnson, Three-level Galerkin methods for parabolic equations, SIAM J. Numer. Anal. 11 (1974), 392-410.

7. G. H. Ganser and D. A. Drew, Nonlinear periodic waves in a two-phase flow model, SIAM J. Appl. Math. 47 (1987), 726-736.

8. __ Nonlinear analysis of a uniform fluidized bed (submitted).

9. M. T. Nakao, Error estimates of a Galerkin method for some nonlinear Sobolev equations in one space dimension, Numer. Math. 47 (1985), 139-157. 
10. L. B. Wahlbin, A dissipative Galerkin method applied to some quasilinear hyperbolic equations, RAIRO Numer. Anal. R-2 (1974), 109-117.

11. __ A dissipative Galerkin method for the numerical solution of first order hyperbolic equations, Mathematical Aspects of Finite Elements in Partial Differential Equations (C. de Boor, ed), Academic Press, New York, 1974, pp. 147-169.

Departments of Mathematics and Engineering Mechanics, University of Kentucky, LEXINGTON, KentUCKY 40506

E-mail address: mat110@ukcc.uky.edu

Departamento de Matematica Aplicada y Computacion, Facultad de Ciencias, UniVERSidAd DE VALladolid, VAlladolid, SPain

E-mail address: sanzserna@cpd.uva.es

Department of Mathematics, West Virginia University, Morgantown, West Virginia 26506

E-mail address: u0acb@wvnvm.bitnet 